\title{
Bronchial compression in an infant with isolated secundum atrial septal defect associated with severe pulmonary arterial hypertension
}

Sung-Hee Park, MD', So Young Park, MD', Nam Kyun Kim, MD', Su-Jin Park, MD', Han Ki Park, MD², Young Hwan Park, MD², Jae Young Choi, $\mathrm{MD}^{1}$

${ }^{1}$ Division of Pediatirc Cardiology, Congenital Heart Disease Center, Severance Cardiovascular Hospital, Department of Pediatrics, Yonsei University Collage of Medicine, Seoul, ${ }^{2}$ Division of Cardiovascular Surgery, Congenital Heart Disease Center, Severance Cardiovascular Hospital, Department of Thoracic and Cardiovascular Surgery, Yonsei University Collage of Medicine, Seoul, Korea

Received: 15 September 2011, Revised: 25 November 2011 Accepted: 31 January 2012

Corresponding author: Jae Young Choi, MD

Division of Pediatirc Cardiology, Congenital Heart Disease Center, Severance Cardiovascular Hospital, Department of Pediatrics, Yonsei University Collage of Medicine, 50 Yonseiro, Seodaemun-gu, Seoul 120-752, Korea

Tel: +82-2-2227-4543, Fax: +82-2-312-9538

E-mail: cjy0122@yuhs.ac

Copyright $@ 2012$ by The Korean Pediatric Society

\begin{abstract}
Symptomatic pulmonary arterial hypertension (PAH) in patients with isolated atrial septal defect (ASD) is rare during infancy. We report a case of isolated ASD with severe PAH in an infant who developed airway obstruction as cardiomegaly progressed. The patient presented with recurrent severe respiratory insufficiency and failure to thrive before the repair of the ASD. Echocardiography confirmed volume overload on the right side of heart and severe PAH (tricuspid regurgitation [TR] with a peak pressure gradient of 55 to $60 \mathrm{mmHg}$ ). The chest radiographs demonstrated severe collapse of both lung fields, and a computed tomography scan showed narrowing of the main bronchus because of an intrinsic cause, as well as a dilated pulmonary artery compressing the main bronchus on the left and the intermediate bronchus on the right. ASD patch closure was performed when the infant was 8 months old. After the repair of the ASD, echocardiography showed improvement of PAH (TR with a peak pressure gradient of 22 to $26 \mathrm{mmHg}$ ), and the patient has not developed recurrent respiratory infections while showing successful catch-up growth. In infants with symptomatic isolated ASD, especially in those with respiratory insufficiency associated with severe $\mathrm{PAH}$, extrinsic airway compression should be considered. Correcting any congenital heart diseases in these patients may improve their symptoms.
\end{abstract}

Key words: Secundum atrial septal defect, Pulmonary arterial hypertension, Airway obstruction, Infant

This is an open-access article distributed under the terms of the Creative Commons Attribution Non-Commercial License (http://creativecommons.org/licenses/by$\mathrm{nc} / 3.0 /$ ) which permits unrestricted non-commercial use, distribution, and reproduction in any medium, provided the original work is properly cited.

\section{Introduction}

Atrial septal defect (ASD) is a common congenital cardiac lesion with an incidence of 6 to $10 \%{ }^{1)}$. Natural history of isolated secundum ASD in infancy and early childhood is generally benign, and presentation of symptoms or pulmonary arterial hypertension (PAH) is unusual at this age. Elective closure of isolated secundum ASD is performed in the fourth or fifth year of life in case of right-sided volume overload, preferably through interventional device closure ${ }^{2}$. However, some patients require closure of ASD much earlier because 
of severe problems, such as failure to thrive, recurrent respiratory infections, or respiratory insufficiency. Sometimes left-to-right shunt can cause airway compression due to a dilated pulmonary artery. We experienced an infantile case in which a patient with an isolated secundum ASD had persistent PAH and collapse of both lungs due to extrinsic compression of both main bronchi by a dilated pulmonary artery and intra-pulmonary bronchial obstruction.

\section{Case report}

A 1-month-old corrected age, male infant weighing $3.4 \mathrm{~kg}$ was transferred to our institute because of respiratory insufficiency. He

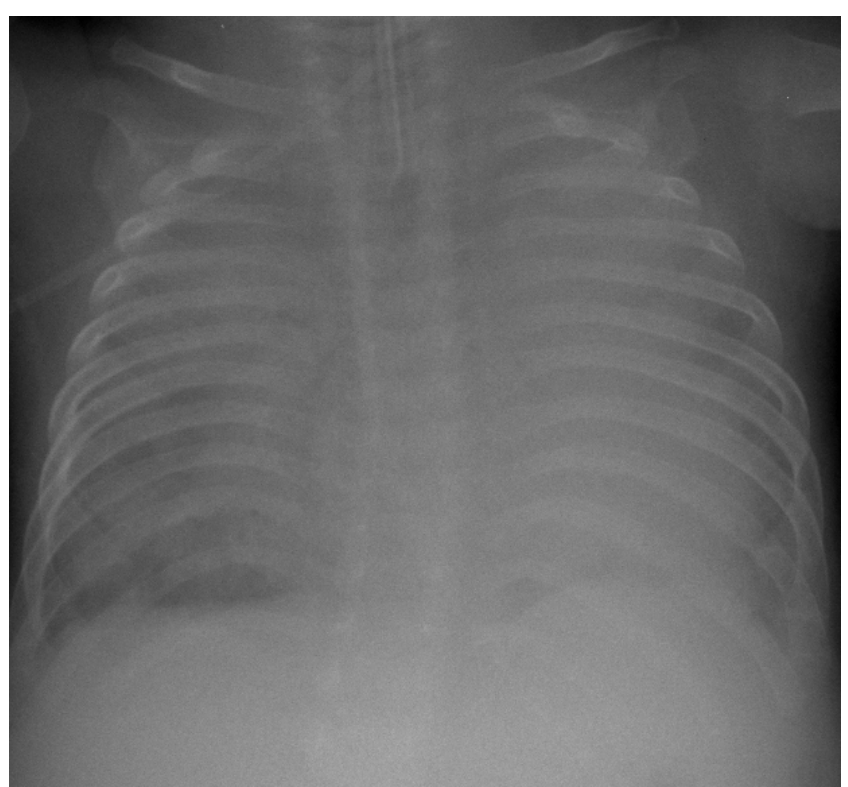

Fig. 1. Chest radiograph at the corrected age of 1 month during the second admission. The patient was intubated, and severe collapse of both lung fields was observed. The diaphragm was flattened, and hyperinflation of both lungs was also observed. was prematurely born by Caesarean section with a birth weight of $1,070 \mathrm{~g}$ at $32^{+1}$ weeks of gestation, and was small for gestational age. The patient was admitted for 50 days including neonatal period and treated with $\mathrm{O} 2$ for the first 4 days after birth due to pneumonia. There was no evidence of hyaline membrane disease or bronchopulmonary dysplasia on chest radiographs and computed tomography (CT) also. On the physical examination, bronchial breathing sounds were increased with rhonci. Regular heart beats were heard with grade 2 systolic murmur on left upper sternal border. He was diagnosed as secundum ASD in the neonatal period. Echocardiography confirmed secundum ASD (diameter 7×12 mm), right-sided volume overload, tricuspid regurgitation (TR) with a peak pressure gradient of 55 to $60 \mathrm{mmHg}$, dilated main pulmonary artery (diameter $19 \mathrm{~mm}$ ) and both pulmonary artery (diameter 11 to 12 $\mathrm{mm}$ ). When the systolic pulmonary artery pressure was estimated from the peak flow velocity in the TR jet by using the modified Bernoulli equation, his pulmonary artery pressure was 58 to 65 mmHg.

At first admission, he was intubated and ventilator care was needed because of progressive respiratory distress. The chest radiographs demonstrated severe collapse of both lung fields at the time (Fig. 1). CT was performed to rule-out other diseases associated with $\mathrm{PAH}$ at the corrected age of 1 month. A CT scan showed a collapse in the dependent portion of the right lung and a total collapse in the lower lobe of the left lung. Narrowing of main bronchus and right intermediate bronchus were evident and an intrinsic cause like bronchomalacia was suspected (Fig. 2). Viral culture studies (respiratory syncytial virus, adenovirus, influenza A virus) were done; results were all negative. After conservative management, he was weaned off the ventilator within 2 weeks. The infant was prescribed with heart failure medications (digoxin $10 \mathrm{mcg} / \mathrm{kg} /$ day divided by 2 ,

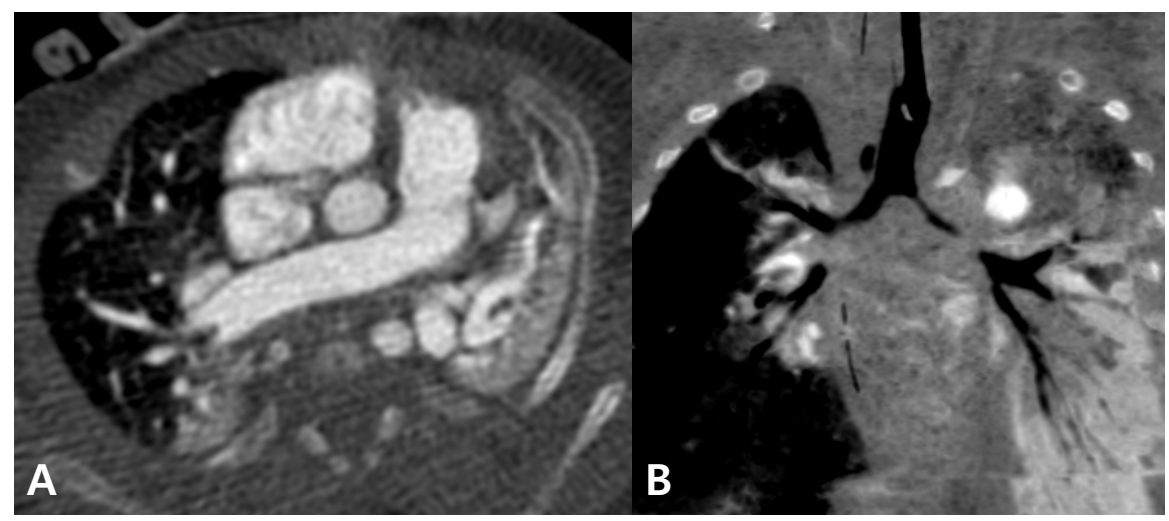

Fig. 2. Computed tomography at the corrected age of 1 month. (A) Collapse in the dependent portion of the right lung and a total collapse in the lower lobe of the left lung are apparent. (B) Coronal image shows narrowing of the main bronchus on the left and the intermediate bronchus on the right. Narrowing of the bronchus was suspected to be because of an intrinsic cause such as bronchomalacia. 


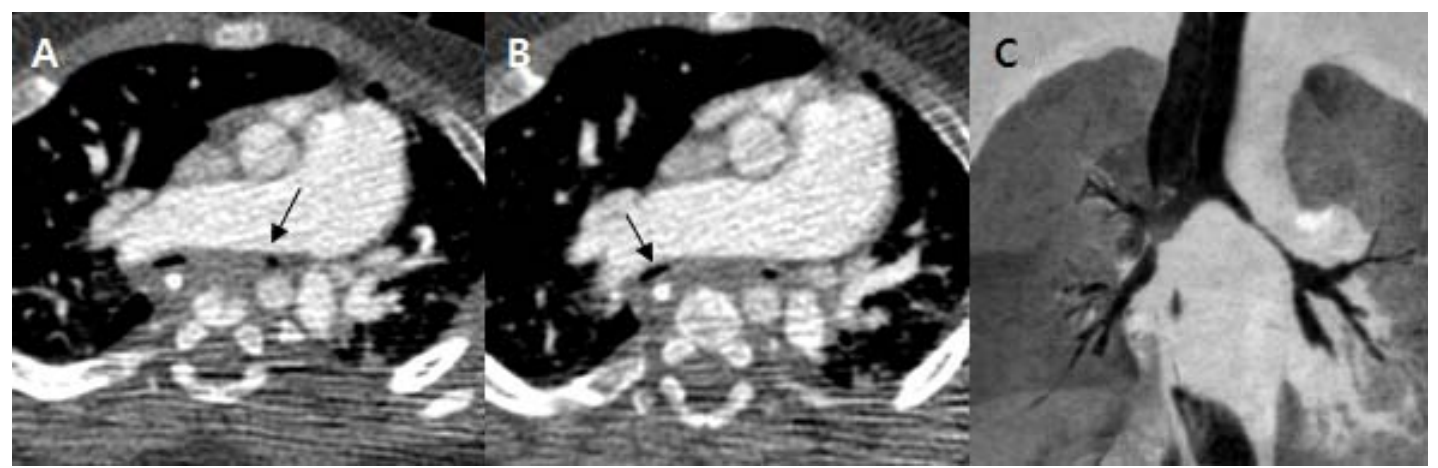

Fig. 3. Preoperative computed tomography at the corrected age of 8 months. Axial image showed dilated pulmonary artery compressing the main bronchus on the left $(A$, arrow) and the intermediate bronchus on the right $(B$, arrow). (C) Coronal image shows narrowing of the main bronchus on the left and the intermediate bronchus on the right side.

furosemide $1 \mathrm{mg} / \mathrm{kg} /$ day divided by 2 , spironolactone $1 \mathrm{mg} / \mathrm{kg} /$ day divided by 2), and extensive physiotherapy of the lung was performed before he was discharged on the 42nd hospital day.

He was hospitalized 3 more times up until the corrected age of 4 months due to recurrent respiratory infection and respiratory distress. We expected catch-up growth and improvement of narrowed airway suspected due to bronchomalasia with patient growth. However, on the 4th hospital admission, he required mechanical ventilation for 40 days due to respiratory insufficiency. Follow-up echocardiograph showed sustained PAH with a TR pressure gradient of $60 \mathrm{mmHg}$. After he was weaned off mechanical ventilation, a follow-up CT scan was performed at the corrected age of 8 months, which revealed dilated pulmonary artery compressing the left main bronchus and right intermediate bronchus, but showed improvement of consolidation in both lungs and mild improvement of stenosis of the right main bronchus (Fig. 3). Up until the corrected age of 8 months the patient was underweight $(5.6 \mathrm{~kg})$ and less than the $3 \mathrm{rd}$ percentile of his age.

To correct these problems, ASD patch closure was performed at this stage. We recognized that he did not suffer from bronchomalacia because his respiratory distress improved after repair of ASD, and the intrinsic cause of airway compression suspected on initial CT scan was due to intra-pulmonary compression resulting from enlarged bronchial vessels and lymphatics. Postoperative echocardiogram showed improvement of TR with decreased peak pressure gradient (22 to $26 \mathrm{mmHg}$ ), immediately after the operation. Postoperative recovery was uneventful and he was extubated on the 3rd postoperative day. He was discharged from the hospital on the 22th postoperative day. The patient is doing well 29 months following surgery and is without any medication. Both lungs appear to be normally expanded, as demonstrated by chest X-ray taken 13 months after the operation (Fig. 4). Postoperative CT was not performed to confirm improvement of bronchial compression, because he did not suffer from any recurrent respiratory infections. At present (3 years

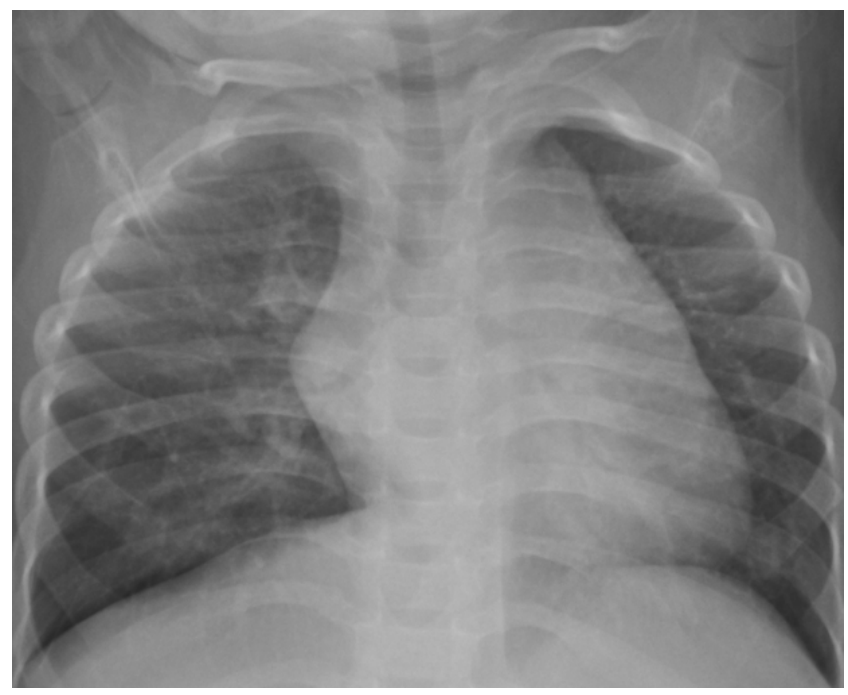

Fig. 4. Postoperative chest radiograph at the corrected age of 22 months. Both the lungs appear to be normally expanded.

and 2 months), he shows catch-up growth with $12 \mathrm{~kg}$ of body weight, which is between $3 \mathrm{rd}$ and 10 th percentile of his age.

\section{Discussion}

Our case reports a rare instance where an infant with isolated ASD presented with severe $\mathrm{PAH}$, and showed symptoms of airway compression. Lammers et al. ${ }^{3)}$ reported that most patients with symptomatic secundum ASD requiring early surgery in the first year of life had ASD as part of syndromatic diseases or malformation complexes, or with trisomy 21 which may be the cause of PAH. In another report, pulmonary vascular obstructive disease was considered to be the primary abnormality in these symptomatic infant with $\mathrm{ASD}^{4)}$. Andrews et al. ${ }^{5)}$ pointed out the need to search carefully for other underlying cardiac or pulmonary abnormalities and recommended closure of the ASD, if other causes for PAH are excluded.

Tracheo-bronchial compression is known to be a cause of 
symptomatic ASD, especially in those who show respiratory insufficiency. Extrinsic airway compression is usually due to a vascular sling, whereas other causes are less common. However, as a result of close proximity of the pulmonary arteries to the bronchi, large leftto-right shunts can compress the airways ${ }^{6}$. Airway compression in the setting of congenital heart disease can result in frequent upper respiratory infection, wheezing, pneumonia, atelectasis and hyperinflation. Wheezing can be the result of intra-luminal bronchial obstruction, interstitial edema, bronchial narrowing and airway hyper-reactivity as well as extrinsic airway compression ${ }^{7}$. Intra-luminal bronchial obstruction can result from enlarged bronchial vessels and lymphatics, both of which can result from increased intracardiac filling pressures ${ }^{6}$. Therefore, extrinsic airway compression and intra-luminal bronchial compression should be considered as causes, in patients with severe respiratory insufficiency or atelectasis following respiratory infection, even if patients have been diagnosed as isolated secundum ASD. For differential diagnosis between extrinsic and intrinsic airway compression, bronchoscopy can be helpful, although it could not be done in this case.

Even a mild degree of extrinsic compression of the bronchus in infants results in airway obstruction because bronchial luminal resistance is inversely proportional to the 4 th power of radius of the lumen $^{8,9)}$. Significantly lower small airway obstruction in children with left-to-right shunts correlated with degrees of $\mathrm{PAH}^{10}$. Prolonged extrinsic compression may result in tracheomalacia, bronchomalasia, or both. Pulmonary artery-induced bronchial compression has also been shown to be an important cause of congenial lobar emphysema ${ }^{11)}$. This patient showed improvement of intrinsic bronchial narrowing. If there was not new development of extrinsic airway compression and failure to thrive, we expected to treat the patient by device closure after more weight had been gained. Some reports suggest that device closure of ASD is an effective and fairly safe alternative to surgery in infants ${ }^{12)}$.

In conclusion, in infants with symptomatic isolated ASD, especially those with respiratory insufficiency associated with $\mathrm{PAH}$, evaluation of extrinsic airway compression should be considered. If extrinsic airway compression is not obvious, the intra-luminal bronchial obstruction should be considered as the cause of intrinsic airway compression. These patients may show symptomatic improvement by correction of their congenital heart disease that causes the left-to-right shunt.

\section{References}

1. Dickinson DF, Arnold R, Wilkinson JL. Congenital heart disease among 160480 liveborn children in Liverpool 1960 to 1969: implications for surgical treatment. Br Heart J 1981;46:55-62.

2. Chan KC, Godman MJ, Walsh K, Wilson N, Redington A, Gibbs JL. Transcatheter closure of atrial septal defect and interatrial communications with a new self expanding nitinol double disc device (Amplatzer septal occluder): multicentre UK experience. Heart 1999;82:300-6.

3. Lammers A, Hager A, Eicken A, Lange R, Hauser M, Hess J. Need for closure of secundum atrial septal defect in infancy. J Thorac Cardiovasc Surg 2005;129:1353-7.

4. Bull C, Deanfield J, de Leval M, Stark J, Taylor JF, Macartney FJ. Correction of isolated secundum atrial septal defect in infancy. Arch Dis Child 1981;56:784-6.

5. Andrews R, Tulloh R, Magee A, Anderson D. Atrial septal defect with failure to thrive in infancy: hidden pulmonary vascular disease? Pediatr Cardiol 2002;23:528-30.

6. Kussman BD, Geva T, McGowan FX. Cardiovascular causes of airway compression. Paediatr Anaesth 2004;14:60-74.

7. Berlinger NT, Long C, Foker J, Lucas RV Jr. Tracheobronchial compression in acyanotic congenital heart disease. Ann Otol Rhinol Laryngol 1983;92(4 Pt 1):387-90.

8. Corno A, Giamberti A, Giannico S, Marino B, Rossi E, Marcelletti C, et al. Airway obstructions associated with congenital heart disease in infancy. J Thorac Cardiovasc Surg 1990;99:1091-8.

9. Sebening C, Jakob H, Tochtermann U, Lange R, Vahl CF, Bodegom $\mathrm{P}$, et al. Vascular tracheobronchial compression syndromes: experience in surgical treatment and literature review. Thorac Cardiovasc Surg 2000;48:164-74.

10. Kussman BD, Geva T, McGowan FX. Cardiovascular causes of airway compression. Paediatr Anaesth 2004;14:60-74.

11. Stanger P, Lucas RV Jr, Edwards JE. Anatomic factors causing respiratory distress in acyanotic congenital cardiac disease. Special reference to bronchial obstruction. Pediatrics 1969;43:760-9.

12. Diab KA, Cao QL, Bacha EA, Hijazi ZM. Device closure of atrial septal defects with the Amplatzer septal occluder: safety and outcome in infants. J Thorac Cardiovasc Surg 2007;134:960-6. 